\title{
Feature Extraction Based on Co-occurrence of Adjacent Local Binary Patterns
}

\author{
Ryusuke Nosaka, Yasuhiro Ohkawa, and Kazuhiro Fukui \\ Graduate School of Systems and Information Engineering, \\ University of Tsukuba, Japan \\ \{nosaka, ohkawa\}@cvlab.cs.tsukuba.ac.jp, kfukui@cs.tsukuba.ac.jp
}

\begin{abstract}
In this paper, we propose a new image feature based on spatial co-occurrence among micropatterns, where each micropattern is represented by a Local Binary Pattern (LBP). In conventional LBP-based features such as LBP histograms, all the LBPs of micropatterns in the image are packed into a single histogram. Doing so discards important information concerning spatial relations among the LBPs, even though they may contain information about the image's global structure. To consider such spatial relations, we measure their co-occurrence among multiple LBPs. The proposed feature is robust against variations in illumination, a feature inherited from the original LBP, and simultaneously retains more detail of image. The significant advantage of the proposed method versus conventional LBP-based features is demonstrated through experimental results of face and texture recognition using public databases.
\end{abstract}

Keywords: Image feature extraction, local binary pattern (LBP), co-occurrence, face recognition, texture recognition.

\section{Introduction}

In this paper, we propose a new Local Binary Pattern (LBP)-based feature by introducing the spatial co-occurrence of adjacent LBPs. LBP-based features, such as LBP histograms, have recently attracted attention as a fundamental technique in the applications of texture recognition, face recognition, and facial expression recognition [1|2 3|4 5, owing to their high robustness to changes in illumination and their efficient computation. The basic idea of the LBP histogram, the focus of this paper, is the representation of entire images as a composition of numerous LBPs, where each LBP is extracted from a local region. LBP was originally designed as a texture description for a local region, called a micropattern 6]. LBP is a binary pattern that represents the magnitude relation between the center pixel of a local region and its neighboring pixels. LBP is obtained by thresholding the image intensity of the surrounding pixels with that of the center pixel. In the LBP histogram, the obtained binary patterns are converted to a decimal number as a label, and a histogram is generated from the labels of all the local regions of the whole image. 
Since LBP considers only the magnitude relation between the center and neighboring pixel intensities, LBP is invariant to uniform changes of image intensity over the entire image, making it robust against changes in illumination. This characteristic of LBP has led it to become a standard feature for face recognition and facial expression analysis [7.

Unfortunately, however, spatial relations among the LBPs are mostly discarded during the LBP histogram generation process, because the LBPs are forcedly packed into a single histogram, resulting in the loss of global image information. This suggests that there is still a room for further improvement to the performance of LBP-based features, while retaining invariance to changes in illumination.

To consider the spatial relation among LBPs, we introduce the concept of cooccurrence. Co-occurrence is often used to extract information related to global structures in various local region-based features, e.g. Co-HOG [8], GLAC 9 ] and Joint Haar-like Features 10. Although co-occurrence of LBPs can be obtained as a heuristic problem, we introduce a more sophisticated way to obtain the co-occurrences of all combinations of LBPs by using auto-correlation matrices calculated from two considered LBPs. The calculation process will show that the proposed feature is a natural extension of the original LBP in that the proposed feature consists of both the original LBP and the co-occurrence of LBPs.

Fig 1 shows the difference between an LBP histogram and the histogram of the spatial co-occurrence between LBPs. The three image examples are composed of three LBP A and three LBP B patterns, as shown in Fig 1(a). Since the number of LBP A and LBP B patterns in each image are the same, the LBP histograms are generated from the three images coincide with each other, as shown in Fig 1(b). In contrast, the histograms of the spatial co-occurrence extracted from each image are quite different, as shown in Fig[1(c). From this
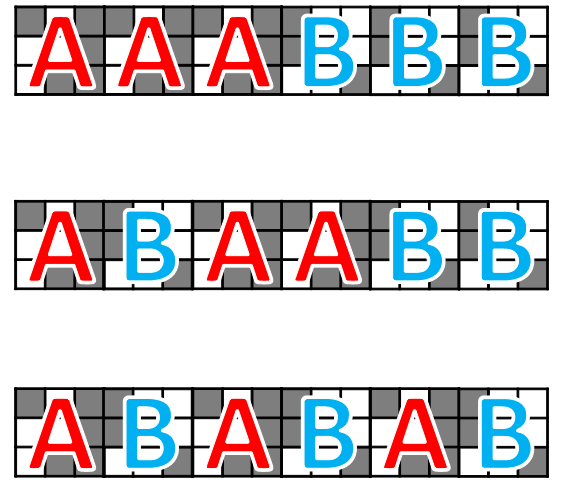

(a) Example images
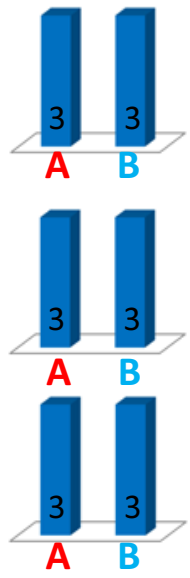

(b) LBP histograms
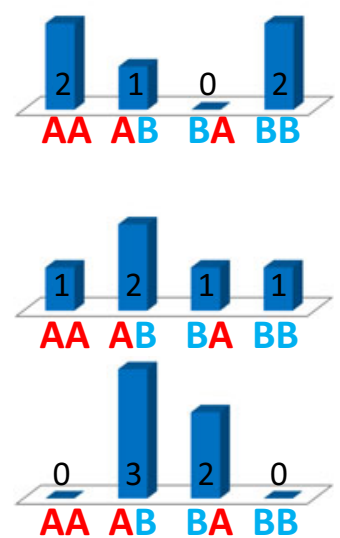

(c) Histograms of the spatial co-occurrence between LBPs

Fig. 1. Difference of LBP histogram and histogram of spatial co-occurrence between LBPs 
simple example, we can see that the expression ability of the original LBP is insufficient, and the spatial co-occurrence of LBPs is a valid requirement for realizing a higher expression ability.

The remainder of this paper is organized as follows. In Section 2, we briefly review the LBP algorithm and extend it by introducing the concept of cooccurrence of adjacent LBPs. Section 3 describes evaluation experiments confirming the validity of the proposed feature. Section 4 presents our conclusions.

\section{The Proposed LBP-Based Image Feature}

\subsection{Local Binary Pattern Histograms}

LBP indicates a magnitude relation between a center pixel and its neighboring pixels in a micropattern. Fig 2 shows an example of a micropattern and an LBP corresponding to it. The LBP ' 10000111 ' is obtained by thresholding $3 \times 3$ neighbor pixels with the value of the center pixel, 5 . The binary pattern is then converted to its decimal equivalent, 135. LBP histograms are generated from the decimal values of all the micropatterns.

Let $I$ be an image intensity and $\boldsymbol{r}=(x, y)^{\top}$ be a position vector in $I$. The LBP $\boldsymbol{b}(\boldsymbol{r})\left(\in \mathbb{R}^{N_{n}}\right)$ is defined as follows:

$$
b_{i}(\boldsymbol{r})=\left\{\begin{array}{l}
1, I(\boldsymbol{r})<I\left(\boldsymbol{r}+\Delta \boldsymbol{s}_{i}\right) \\
0, \text { otherwise }
\end{array} \quad,\left(i=1, \cdots, N_{n}\right),\right.
$$

where $N_{n}$ is the number of neighbor pixels and $\Delta \boldsymbol{s}_{i}$ are displacement vectors from the position of center pixel $\boldsymbol{r}$ to neighbor pixels. In the original LBP, these parameters are set as follows: $N_{n}=8, \Delta \boldsymbol{s}_{i} \in\left\{( \pm \Delta s, \pm \Delta s)^{\top},( \pm \Delta s, \mp \Delta s)^{\top}\right.$, $\left.( \pm \Delta s, 0)^{\top},(0, \pm \Delta s)^{\top}\right\}, \Delta s=1$. Next, the LBP $\boldsymbol{b}(\boldsymbol{r})$ is converted to a decimal number. Finally, the histogram of the LBPs is generated by considering the decimals as labels.

The magnitude relation of intensities is invariant for change such as scalar times intensity in an entire image. In other cases of illumination conditions, the magnitude relation is also robust. Therefore, LBP is robust against illumination variance.

\subsection{Co-occurrence of Adjacent Local Binary Patterns}

The co-occurrence of adjacent LBPs is defined as an index of how often their combination occurs in the whole image. Here, we explain how to calculate the co-occurrence of LBPs.

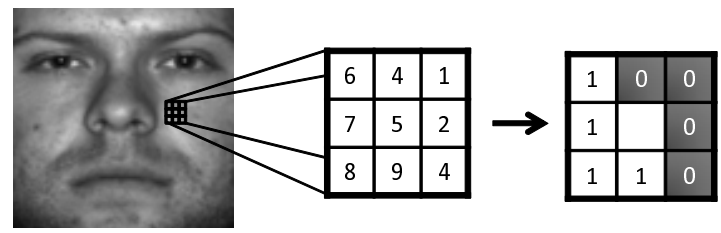

$(10000111)_{2}$

$=135$

Fig. 2. Original LBP 
Fig 3 shows a comparison of the original LBP and the co-occurrence of LBPs. The original LBP shown in Fig [3(a) can represent only a simple image pattern.On the other hand, the combination of multiple LBPs can represent various image patterns derived from more complicated surfaces, as shown in Fig 3(b).

In the proposed feature, the number of possible combinations of LBPs is significantly greater than that of the original LBPs. It is therefore difficult to use a rule-based program to compute the co-occurrence of all combinations when there are many types of LBPs. Instead of using a heuristic program, we introduce an auto-correlation matrix as an effective method of calculating the co-occurrence of LBPs. First, although the original LBP uses eight neighbor pixels of a given center pixel, we modify the LBP configuration to consider two sparser configurations, thereby reducing computational cost. One configuration is $\operatorname{LBP}(+)$, which considers only two horizontal and two vertical pixels, as shown in Fig 4(a). The other configuration is the $\operatorname{LBP}(\times)$, which considers the four diagonal pixels shown in Fig $4(\mathrm{~b})$. In the $\operatorname{LBP}(+)$, the parameters are set as follow: $N_{n}=4$, $\boldsymbol{s}_{i} \in\left\{( \pm \Delta s, 0)^{\top},(0, \pm \Delta s)^{\top}\right\}$. In the $\operatorname{LBP}(\times)$, the parameters are set as follow: $N_{n}=4, \boldsymbol{s}_{i} \in\left\{( \pm \Delta s, \pm \Delta s)^{\top},( \pm \Delta s, \mp \Delta s)^{\top}\right\}$.

Next, in order to effectively calculate the co-occurrence of LBPs, each LBP is converted to vector $\boldsymbol{f}\left(\in \mathbb{R}^{N_{p}}\right)$, which is defined as follows:

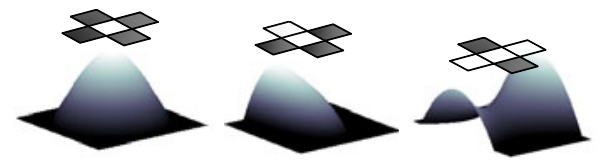

(a) Single LBPs

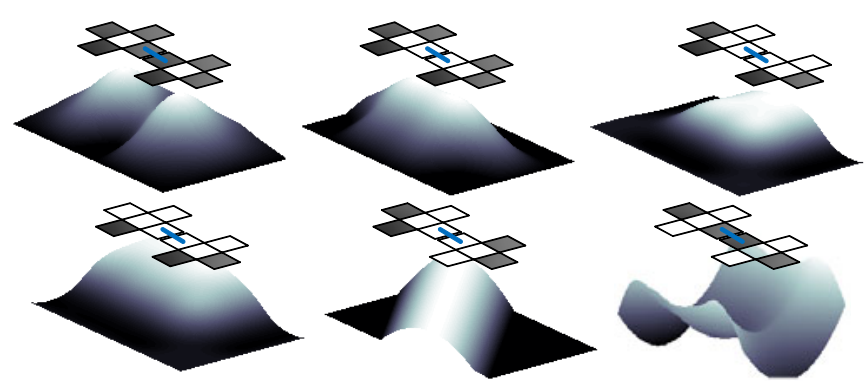

(b) Multiple LBPs

Fig. 3. Comparison of single and multiple LBPs

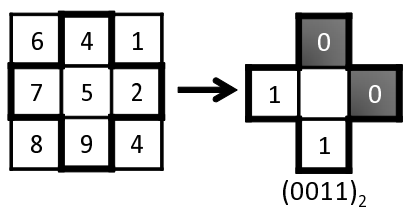

(a) $\operatorname{LBP}(+)$

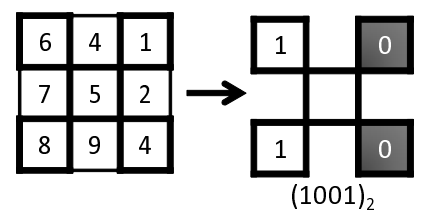

(b) $\operatorname{LBP}(x)$

Fig. 4. Sparse LBPs 


$$
f_{i}(\boldsymbol{r})=\delta_{i, l(\boldsymbol{b}(\boldsymbol{r}))},
$$

where, $N_{p}$ is the number of all the possible LBPs, $N_{p}=2^{N_{n}}$ for example, in the original LBP, $N_{p}=2^{8}=256, N_{n}$ is the number of neighbor pixels, $\delta_{i, j}$ is Kronecher's delta, and $l(\boldsymbol{b}(\boldsymbol{r}))$ is the label of $\boldsymbol{b}(\boldsymbol{r})$.

To effectively calculate the co-occurrence of LBPs, we consider the $N_{p} \times N_{p}$ auto-correlation matrix defined by the following equation:

$$
\boldsymbol{H}(\boldsymbol{a})=\sum_{\boldsymbol{r} \in I} \boldsymbol{f}(\boldsymbol{r}) \boldsymbol{f}(\boldsymbol{r}+\boldsymbol{a})^{\top},
$$

where $\boldsymbol{a}$ is the displacement vector from the reference LBP to its neighbor LBP. The element $H_{i, j}(\boldsymbol{a})$ of Eq. (3) indicates the number of pairs of adjacent LBP $i$ LBP $j$. After shift-equivalent patterns are removed, $\boldsymbol{a}$ is set as follows: $\left\{(\Delta r, 0)^{\top}\right.$, $\left.(\Delta r, \Delta r)^{\top},(0, \Delta r)^{\top},(-\Delta r, \Delta r)^{\top}\right\}$. Fig 5 shows all the configuration patterns of $\boldsymbol{r}$ and $\boldsymbol{r}+\boldsymbol{a}$ in Eq. (3).

Next, we explain characteristics of the proposed feature. Although the proposed feature has high dimensionality $\left(4 N_{p}{ }^{2}\right)$, the computational cost is low due to the sparsity of the LBP. The original LBP histogram can be obtained as the summation of the column vectors of the matrix $\boldsymbol{H}(\boldsymbol{a})$ defined by Eq.(3). This means that the proposed feature retains the original LBPs along with cooccurrence information, making it a natural extension of the original LBP.

Fig 6 shows the flow of extracting the proposed feature from an image. The example image has four LBPs (Fig 6(a)). The labels of these LBPs are 2, 8, 9, and 14, respectively. In the case of the displacement vector $\boldsymbol{a}=(\Delta r, 0)^{\top}$, there are two LBP pairs ( upper left, upper right\} and \{lower left, lower right\}) in the image. Since the labels are $(2,14)$ and $(8,9)$, the elements corresponding to these labels in Eq.(3) are set to 1 and other elements are set to 0. For other displacement vectors: $\boldsymbol{a}=(0, \Delta r)^{\top},(\Delta r, \Delta r)^{\top}$ and $(-\Delta r, \Delta r)^{\top}$, an auto-correlation matrix $\boldsymbol{H}(\boldsymbol{a})$ is similarly generated as shown in Fig, 6 (b).

Fig [7 shows the process flow of proposed feature. Firstly, LBPs are extracted from the input image as shown in Fig:7(a). Next, we compute four $N_{p} \times N_{p}$ auto-correlation matrices of spacial co-occurrences of adjacent $\operatorname{LBPs}, \boldsymbol{H}(\boldsymbol{a})$, as shown in Fig:7(b). Finally, these matrices are vectorized and combined to a $4 N_{p}^{2}$-dimensional feature vector $\boldsymbol{z}($ Fig $7(\mathrm{c}))$.

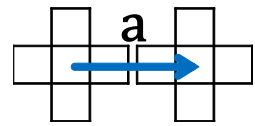

$(\Delta r, 0)$

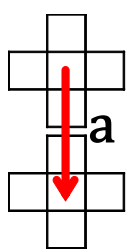

$(0, \Delta r)$

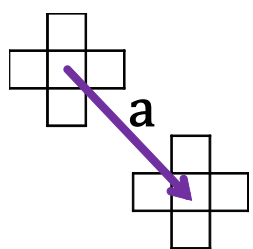

$(\Delta r, \Delta r)$

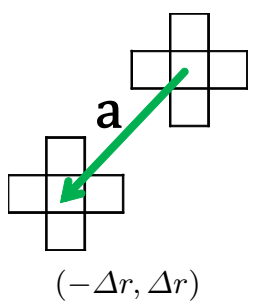

$(-\Delta r, \Delta r)$

Fig. 5. Configuration patterns of proposed feature 


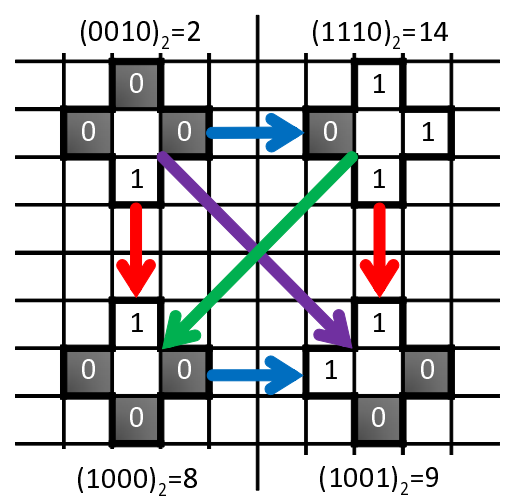

(a) Example image

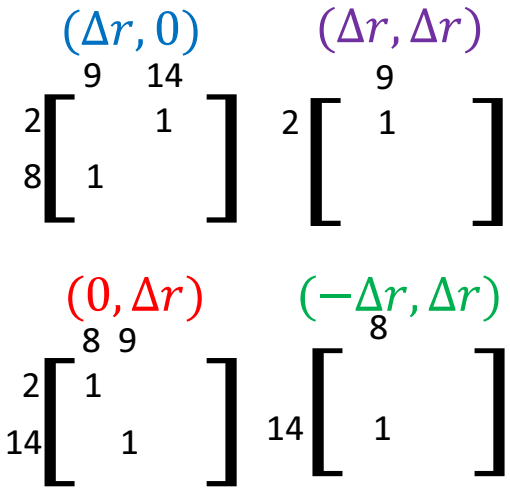

(b) Auto-correlation matrices of co-occurrence of LBPs, $\boldsymbol{H}(\boldsymbol{a})$

Fig. 6. Example of obtaining proposed feature

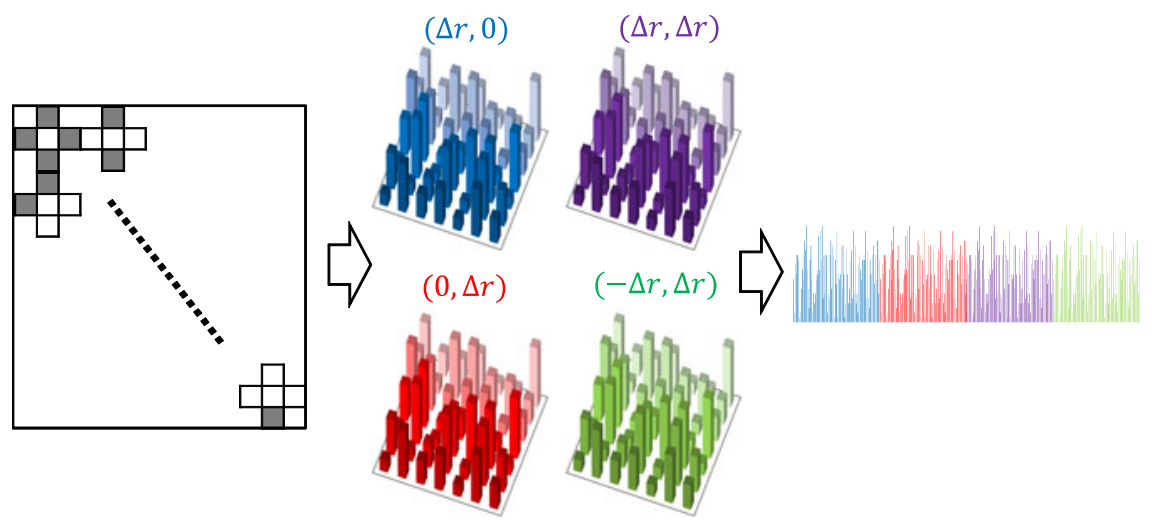

(a) Extracting LBPs from the image (b) Auto-correlation matrices of co-occurrence of LBPs, $\boldsymbol{H}(\boldsymbol{a})$ (c) Combined feature $\boldsymbol{z}$

Fig. 7. Process flow of proposed feature

\section{Experiments and Considerations}

To confirm the proposed method's validity, we applied it to face recognition and texture recognition tasks.

\subsection{Face Recognition Experiment}

Setup. In this experiment, we evaluated the proposed feature using the Extended Yale Face Database B [1]. Fig 8 shows examples of images included in the dataset. The dataset contains the faces of 38 subjects under 64 variations in 

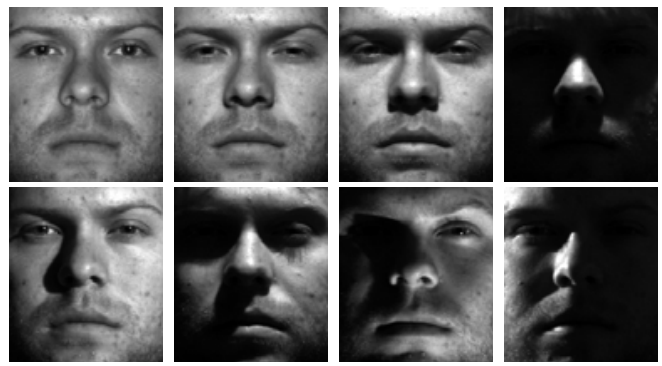

Fig. 8. Examples from the Extended Yale Face Database B

illumination for each. All images are frontal face images cropped to $168 \times 168$ pixels. We resized the images to $88 \times 88$ pixels for this experiment. Images with frontal lighting were used as a training set (one image per person). The remaining images were used as a testing set (63 images per person).

We compared the proposed feature with a raw image feature, a Gabor image feature [12], and the LBP histogram. The raw image feature was obtained by vectorizing the input image.

For the LBP histogram we prepared three types of features, differing in the selection of surrounding pixels: $3 \times 3$ neighbor pixels (original $\mathrm{LBP}$ ), $\operatorname{LBP}(+)$ and $\operatorname{LBP}(\times)$. For the proposed feature, we prepared two types of features, differing in the selection of surrounding pixels: $\operatorname{LBP}(+)$ and $\operatorname{LBP}(\times)$.

The parameters of the proposed feature were changed as follows: $\Delta s=1, \cdots, 5$ and $\Delta r=1, \cdots, 20$. The best correct rate among the results was regarded as the final result.

The image was divided into multiple subregions. Four types of divisions $(1 \times 1$, $2 \times 2,4 \times 4$, and $8 \times 8$ ) were performed. The features extracted from these subregions were integrated into a final feature $\boldsymbol{z}$. Therefore, for each division, the dimension of the final proposed feature $\boldsymbol{z}$ is $4 N_{p}^{2} \times 1,4 N_{p}^{2} \times 2^{2}, 4 N_{p}^{2} \times 4^{2}, 4 N_{p}^{2} \times 8^{2}$, respectively. The region division described above was not performed for the raw image feature and the Gabor image feature.

The nearest neighbor method with $L_{1}$ norm was used as a classifier. The $L_{1}$ norm is usually used as the similarity between two histograms [13, since it has a similar characteristic to the histogram intersection defined by $S\left(\boldsymbol{H}_{1}, \boldsymbol{H}_{2}\right)=$ $\sum_{i} \min \left(H_{1 i}, H_{2 i}\right)$.

Results. Fig 9 shows the results of the experiment. From these results, we can confirm the LBP-based feature's robustness against variations in illumination. In contrast, performances of the raw image feature and the Gabor image feature were poor, due to their sensitivity to illumination. Their performances could not be improved even when considering the co-occurrence.

The results show that the histogram feature of LBPs outperform other features. In particular, the performance of the proposed feature with co-occurrence of LBPs is remarkable. It has achieved the best performance using parameters $\Delta s=1, \Delta r=3$, and $8 \times 8$ division. We can see that increasing number of division increases the performance due to keeping spacial information. 


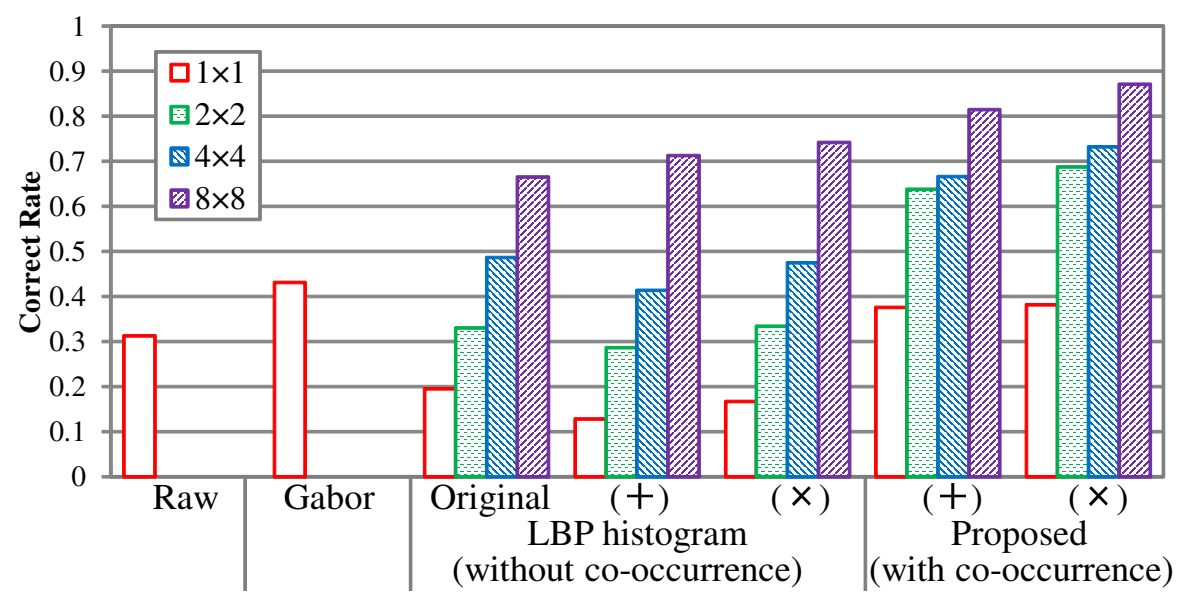

Fig. 9. Results of face recognition experiment

Moreover, we can also see that the performance of the proposed feature depends on the configuration of LBP. When the division number was set to a large value, the performances of both the $\operatorname{LBP}(+)$ and $\operatorname{LBP}(\times)$ were superior to the original LBP. The reason for this result can be explained as follows. The dimension of the original LBP feature is higher than that of the $\operatorname{LBP}(+)$ and $\operatorname{LBP}(\times)$. Therefore, the distribution of the original LBPs tends to be too sparse and unstable as the division number increases.

\subsection{Texture Recognition Experiment}

Setup. In this experiment, we evaluated the proposed method using Outex_TC from the public database Outex [14. Table 1 shows the details of the dataset. Fig[10 shows examples of images from the dataset.

Outex_TC_00000 - 00002 contains grayscale images of 24 textures at different sizes. Outex_TC_00016 contains 319 textures. The average image intensity value is normalized to 128 , with a standard deviation of 20 .

The images were randomly split between training and testing sets. This division was repeated 100 times to produce 100 evaluation sets. The average of all correct rates over 100 iterations was defined as the final rate.

The proposed feature employed $\Delta s=1, \cdots, 3$ and $\Delta r=1, \cdots, 5$, and the best correct rates were used as the reported results. The dimension of the final

Table 1. Outex database details

\begin{tabular}{|c|c|c|c|}
\hline Outex ID & Classes & Image sizes & Training/Testing images \\
\hline Outex_TC_00000 & 24 & $128 \times 128$ & 10 \\
Outex_TC_00001 & 24 & $64 \times 64$ & 44 \\
Outex_TC_00002 & 24 & $32 \times 32$ & 184 \\
Outex_TC_00016 & 319 & $128 \times 128$ & 10 \\
\hline
\end{tabular}



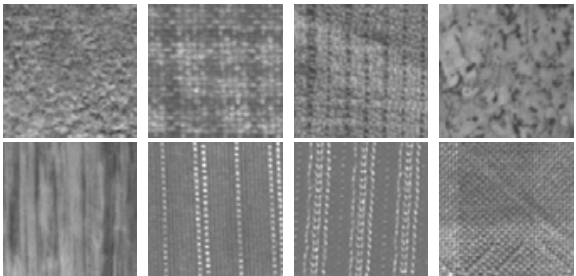

(a) Outex_TC_00000 - 00002
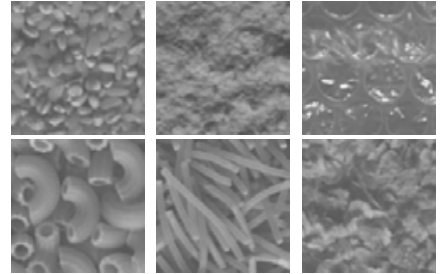

(b) Outex_TC_00016

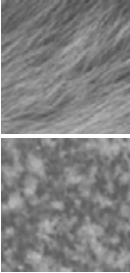

Fig. 10. Outex database examples

Table 2. Results of the texture recognition experiment

\begin{tabular}{|c||ccc|cc|}
\hline \multicolumn{1}{|c||}{} & \multicolumn{3}{c|}{ LBP histogram } & \multicolumn{2}{c|}{ Proposed } \\
\cline { 2 - 6 } Outex ID & Original & $(+)$ & $(\times)$ & $(+)$ & $(\times)$ \\
\cline { 2 - 6 } Outex_TC_00000 & 0.996 & 0.986 & 0.989 & $\mathbf{0 . 9 9 9}$ & $\mathbf{0 . 9 9 9}$ \\
Outex_TC_00001 & 0.985 & 0.930 & 0.948 & $\mathbf{0 . 9 8 9}$ & $\mathbf{0 . 9 8 9}$ \\
Outex_TC_00002 & 0.871 & 0.721 & 0.742 & 0.906 & $\mathbf{0 . 9 1 5}$ \\
Outex_TC_00016 & 0.783 & 0.686 & 0.708 & $\mathbf{0 . 8 3 0}$ & 0.820 \\
\hline
\end{tabular}

proposed feature $\boldsymbol{z}$ is $4 N_{p}^{2}$. The $L_{1}$ nearest neighbor method was used as a classifier.

Results. Table 2 shows the results of the experiment. The results confirm a significant advantage of the proposed feature against the LBP histogram features, which are not considering the co-occurrence. The proposed feature with parameters $\Delta s=1, \Delta r=2$ achieved the best performance among all the features.

In contrast to the previous experiment, the original LBP was better than the $\operatorname{LBP}(+)$ and $\operatorname{LBP}(\times)$ in the case that co-occurrence was not considered. This is because the subregion size used in this experiment was better suited to the original LBP, as compared with other LBPs.

\section{Conclusion}

We have proposed a novel image feature based on the spatial co-occurrence of micropatterns, which are represented by Local Binary Pattern (LBP). The conventional LBP-based features as represented by the LBP histogram still has room for performance improvements. In particular, expression ability for a given image can be improved, since all LBPs are simply summed into a single histogram, thereby discarding spatial relations among the LBPs and the rich image information they contain. To improve their performance, we introduced the extension of original LBP by considering the co-occurrence of adjacent LBPs, measuring cooccurrence with an auto-correlation matrix generated from multiple LBPs. The proposed feature is robust against variations in illumination, because it only depends on the magnitude relation between a center pixel and its surrounding pixels. Experimental results of face and texture recognition tasks using public 
databases have demonstrated a significant advantage of the proposed feature against conventional LBP-based features.

\section{References}

1. Ojala, T., Pietikäinen, M., Mäenpää, T.: Multiresolution gray-scale and rotation invariant texture classification with local binary patterns. IEEE Transactions on Pattern Analysis and Machine Intelligence 24, 971-987 (2002)

2. Zhang, W., Shan, S., Gao, W., Chen, X., Zhang, H.: Local Gabor Binary Pattern Histogram Sequence (LGBPHS): A Novel Non-Statistical Model for Face Representation and Recognition. In: Proc. IEEE International Conference on Computer Vision, vol. 1, pp. 786-791 (2005)

3. Zhao, G., Pietikäinen, M.: Dynamic Texture Recognition Using Local Binary Patterns with an Application to Facial Expressions. IEEE Transactions on Pattern Analysis and Machine Intelligence, 915-928 (2007)

4. Lei, Z., Liao, S., He, R., Pietikainen, M., Li, S.: Gabor volume based local binary pattern for face representation and recognition. In: Proc. IEEE Conference on Automatic Face and Gesture Recognition, pp. 1-6 (2008)

5. Zhang, B., Gao, Y., Zhao, S., Liu, J.: Local derivative pattern versus local binary pattern: face recognition with high-order local pattern descriptor. IEEE Transactions on Image Processing 19, 533-544 (2010)

6. Ojala, T., Pietikäinen, M., Harwood, D.: A comparative study of texture measures with classification based on featured distributions. Pattern Recognition 29, 51-59 (1996)

7. Ahonen, T., Hadid, A., Pietikäinen, M.: Face description with local binary patterns: Application to face recognition. IEEE Transactions on Pattern Analysis and Machine Intelligence 28, 2037-2041 (2006)

8. Watanabe, T., Ito, S., Yokoi, K.: Co-occurrence Histograms of Oriented Gradients for Pedestrian Detection. In: Proc. the 3rd IEEE Pacific-Rim Symposium on Image and Video Technology, pp. 37-47 (2009)

9. Kobayashi, T., Otsu, N.: Image Feature Extraction Using Gradient Local AutoCorrelations. In: Forsyth, D., Torr, P., Zisserman, A. (eds.) ECCV 2008, Part I. LNCS, vol. 5302, pp. 346-358. Springer, Heidelberg (2008)

10. Mita, T., Kaneko, T., Stenger, B., Hori, O.: Discriminative feature co-occurrence selection for object detection. IEEE Transactions on Pattern Analysis and Machine Intelligence 30, 1257-1269 (2008)

11. Lee, K., Ho, J.: Acquiring linear subspaces for face recognition under variable lighting. IEEE Transactions on Pattern Analysis and Machine Intelligence 27, 684698 (2005)

12. Liu, C., Wechsler, H.: Gabor feature based classification using the enhanced fisher linear discriminant model for face recognition. IEEE Transactions on Image Processing 11, 467-476 (2002)

13. Swain Jr., M., Ballard, D.: Color indexing. International Journal of Computer Vision 7, 11-32 (1991)

14. Ojala, T., Mäenpää, T., Pietikäinen, M., Viertola, J., Kyllönen, J., Huovinen, S.: Outex - new framework for empirical evaluation of texture analysis algorithms. In: Proc. IEEE International Conference on Pattern Recognition, pp. 701-706 (2002) 